\title{
Modelo matemático en el currículo del área agrícola en colegios agropecuarios
}

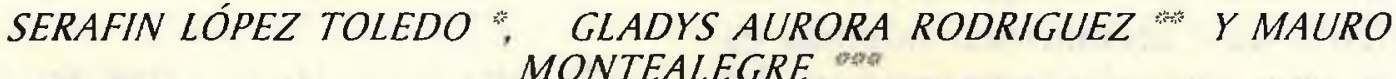

\section{Resumen}

E ste trabajo contiene un modelo matemático aplicable a la produc ción agrícola que relaciona los conceptos de las áreas agrícolas y matemáticas, de tal manera que se complementan y se sirven de apoyo para el desarrollo de la planeación del cultivo, proceso de investigación en producción y mercadeo de los productos. Se fundamenta en la concepción sistémica de la educación y en la necesidad de evolución del campo teniendo como foco de proyección la Institución educativa de carácter técnico de la región.

La Matemática en los Colegios Agropecuarios sirve como herramienta para el desarrollo técnico y científico del campo, a la vez aporta modelos para ser analizados en el aula de clase y extrapolados como resultados para su aplicación en el proceso productivo.

El modelo expuesto es una construcción que coordina los conceptos de: Aritmética, Contabilidad, Estadística, Trigonometría, Análisis Matemático y los procesos de planeación, producción, mercadeo e investigación de plagas.

\section{Introducción}

La matemática se empezó a construir en el mundo real y a él debe volver con sus beneficios. Por esta sencilla razón se ha hecho un esfuerzo por buscar el acercamiento hacia el área agrícola para que sean desarrollados y aplicados los conocimientos de manera creativa.

* Especialista en Educación Matemática con énfasis en la Aplicación de los Sistemas Dinámicos, profesor del Colegio Nacional Antonio Baraya, de Baraya Huila.

Especialista en Educación Matemática con énfasis en la Aplicación de los Sistemas Dinámicos, profesor del Nùcleo Escolar La Arcadia, de Algeciras Huila.

纹解 PHD en Sistemas Dinámicos, profesor de la Universidad Surcolombiana. 
UnIVERSIDAD SURCOLOMBIANA

Este trabajo propone que las Instituciones Educativas desarrollen las áreas como partes estructurales y dinámicas de un sistema, o una red compleja, que es controlado por otros como el económico, social y cultural, globalizados en el macrosistema ambiental.

En el desarrollo del modelo se encuentra el proceso de producción agrícola desglosado en sus distintas fases y parámetros que aparecen para orientar la elaboración y enriquecimiento del currículo de matemáticas. Además es un apoyo pedagógico para recoger del medio la información mediante estadísticas y registros contables con base en el estudio de casos del comportamiento de plagas, enfermedades, infestación de malezas, de pesticidas, abonos y fertilizantes, variaciones climáticas, cambios en el mercado y maximización del beneficio de un recurso.

La idea es que se amplíe estas propuestas a otras áreas del conocimiento y la institución se proyecte a la comunidad con acciones interdisciplinarias que renueven la educación para favorecer la calidad de vida del sector campesino colombiano.

\section{Propuesta}

Partiendo de la Teoría General de Sistemas, y, la concepción de la Teoría de la Complejidad, con su enfoque abarcador y estructural se establece un marco grande donde se desarrolla el modelo: EL SISTEMA AMBIENTAL, donde se da todo proceso y toda transformación de la realidad social, económica y cultural. Ver figura 1.

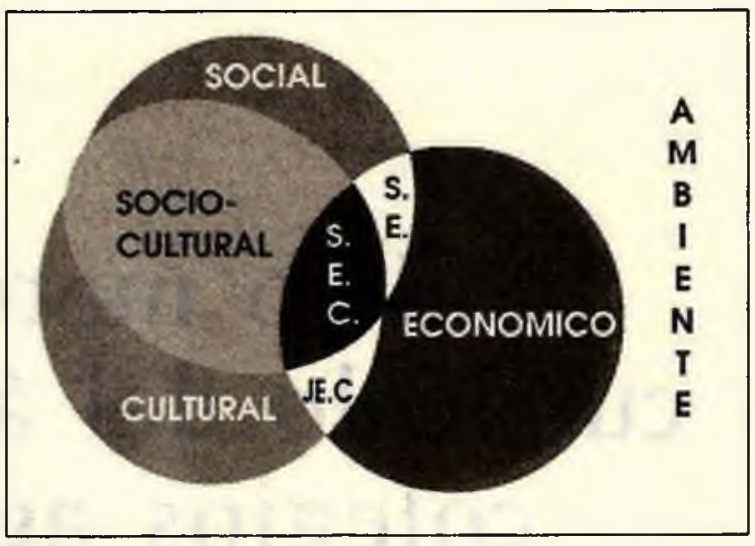

FIGURA 1. Interacción entre los sistemas

"A este nivel las ciencias agrícolas pueden convertirse en una asignatura que se estudiará en el marco de la enseñanza interdisciplinaria"1, las clases de ciencias y matemáticas desarrolladas directamente con situaciones problemáticas surgidas en el contexto de la agricultura facilitará la integración de los conocimientos y la comprensión del sistema productivo,como se sintetiza en la figura 2.

Las principales características de un sistema se denominan variables, parámetros, relaciones y procesos que en este caso involucran: insumos, procesos, producto, mecanismos de control y restricciones. Dichas características describen el sistema y las relaciones entre ellos definen las funciones de las partes y establecen los procedimientos de desarrollo del todo con sus partes. Así en el sistema de producción agrícola existen parámetros que interactúan con el tiempo en el proceso. Claro está que los subprocesos tienen tiempos variados dependiendo de factores ambientales y de manejo del cultivo.

1. UNESCO. La educación en el mundo real. París, 1974.30p. 
PaIdela

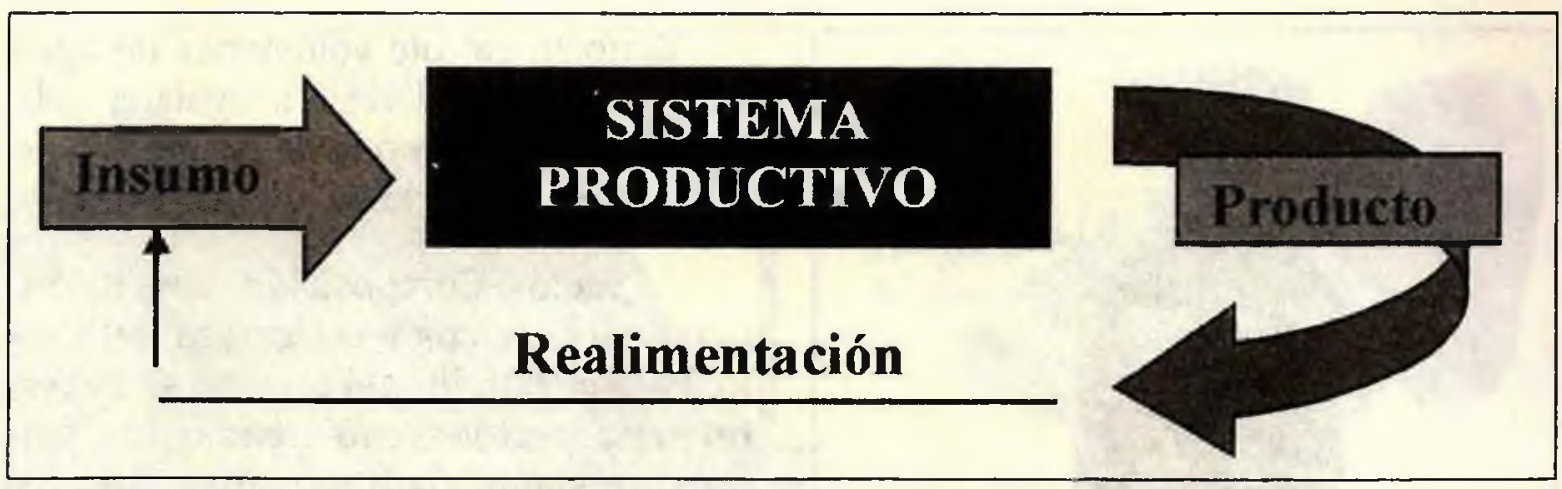

FIGURA 2. Dinámica del sistema de producción: muestra la acción del insumo sobre el sistema productivo y el efecto que produce sobre el.

Para la estructuración del currículo de matemáticas es necesario interrelacionar el tipo de cultivo según la zona y el periodo de producción, dado que las instituciones agrícolas se encuentran en distintos climas (ver diagrama).

Basados en los principales cultivos de la zona donde está la institución se sacan las variables, relaciones y parámetros del proceso productivo para construir el currículo de agrícolas que se tomará como apoyo para elaborar el currículo de matemáticas.
A continuación se presenta una síntesis de los aspectos importantes a tener en cuenta según el periodo de cosecha.

\subsection{Cultivos temporales:}

3.1.1 Variable ambiental. Son parámetros relevantes:

- factor climático: Lluviosidad, altitud y variaciones de temperatura. Para estudiar estos temas es necesario revisar los sistemas de medida, producto cartesiano, funciones y fracciones (figura 3).

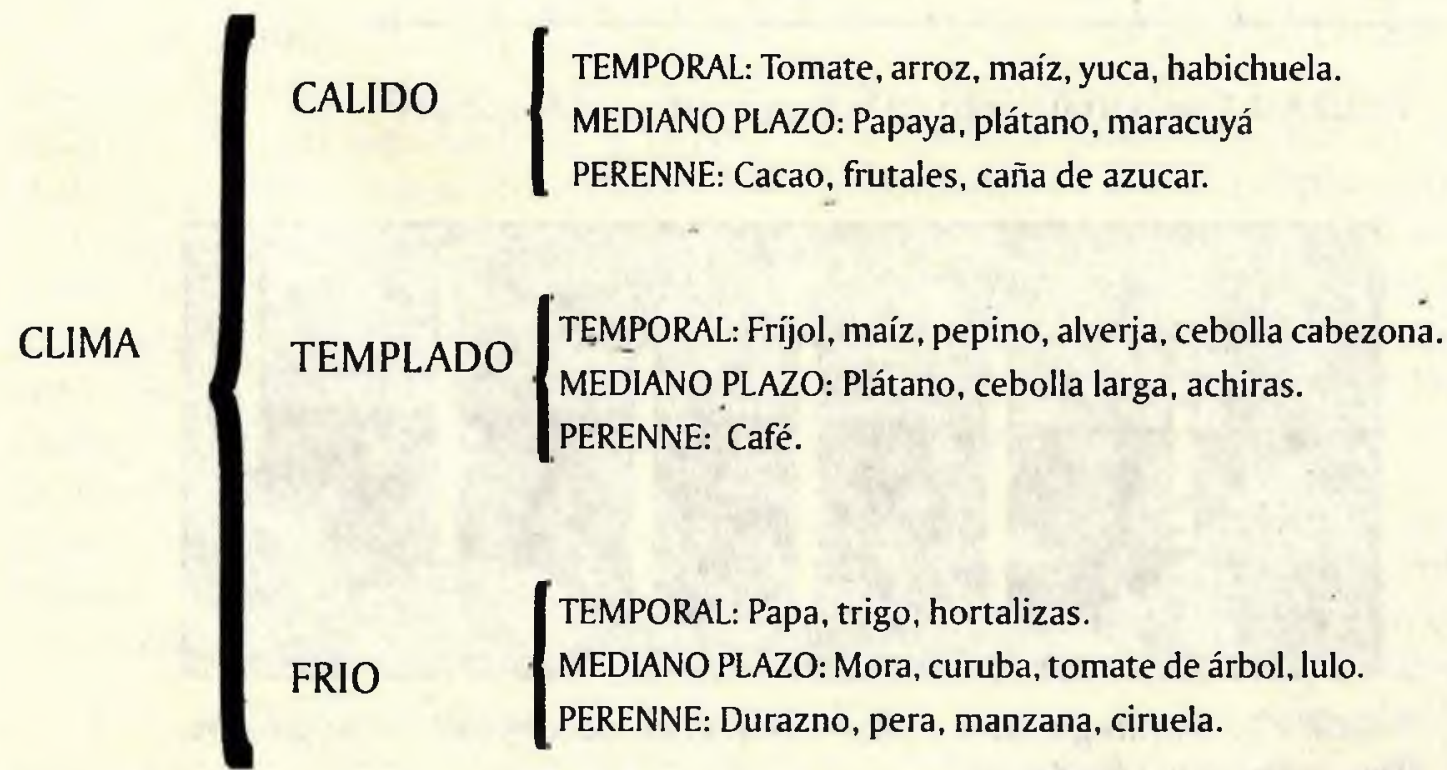


UNIVERSIDAD SURCOLOMBIANA

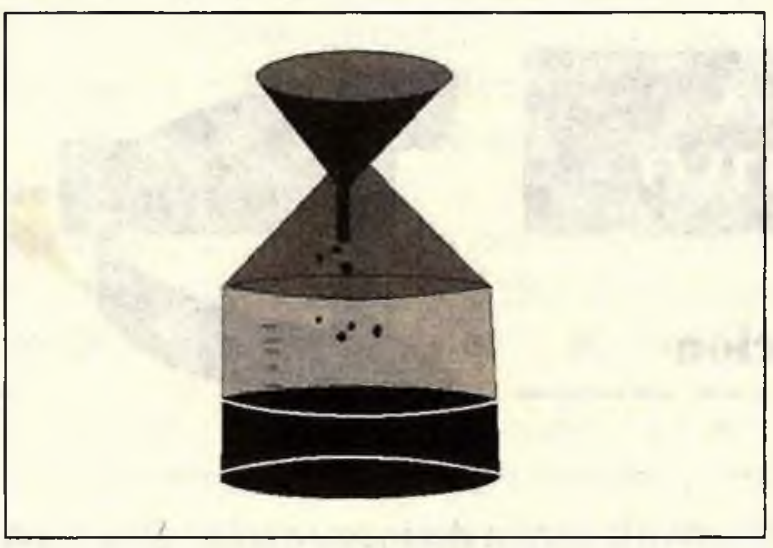

FIGURA 3. Medida del volumen de lluvias con el uso del pluviómetro.

Como estrategias metodológicas se podrían construir modelos sacados de cada situación analizada como por ejemplo las variaciones de la temperatura.
También calcule volúmenes de agua en depósitos regulares e irregulares aplicando técnicas como la de promedios de profundidad y anchura. (figura 5).

- Suelos: Composición, dimensión, pendiente, cercos y estructura del suelo. Para el estudio del terreno se deben revisar aspectos como pendientes, plano cartesiano, figuras planas (áreas y perímetros), volúmenes, máximos y mínimos (maximizar áreas con el mínimo de perímetro para construcción de cercas), proporcionalidad (escala) y nociones de estadística.

Como herramientas de trabajo se pueden usar el teodolito, nivel de mano, la mangueras de nivel y la cinta métrica. (figura 6).

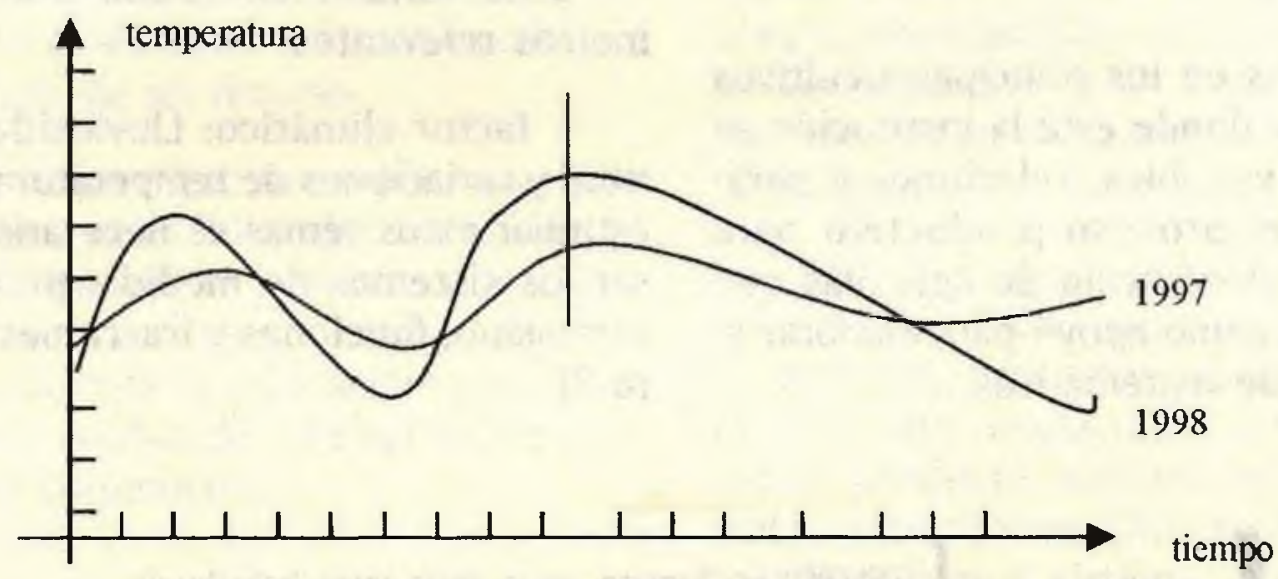

FIGURA 4. Comportamiento de la temperatura en el tiempo.

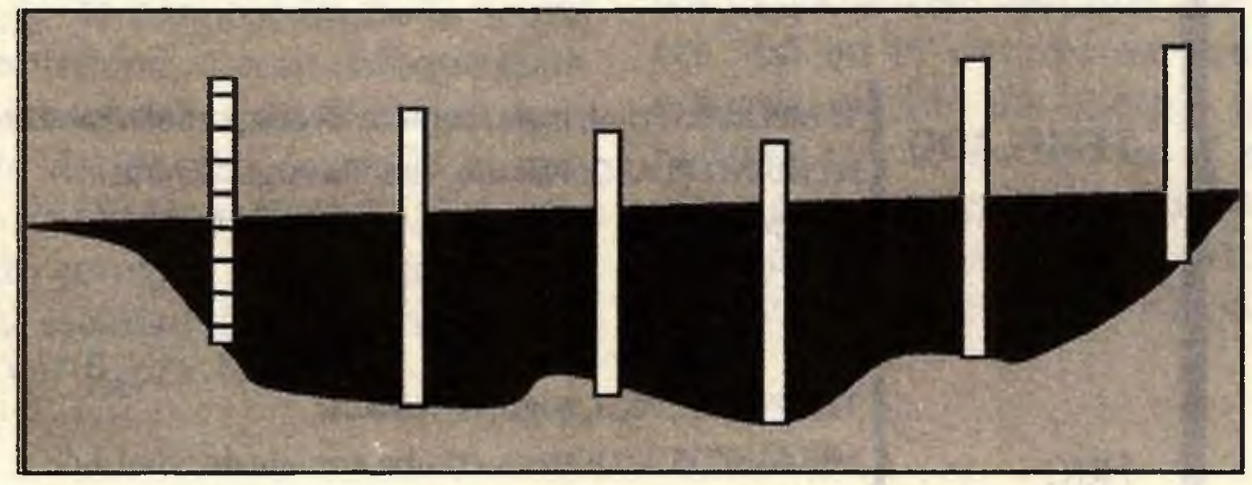

FIGURA 5. Estrategia para hallar volúmenes de depósitos irregulares. Promedio de profundidad. 

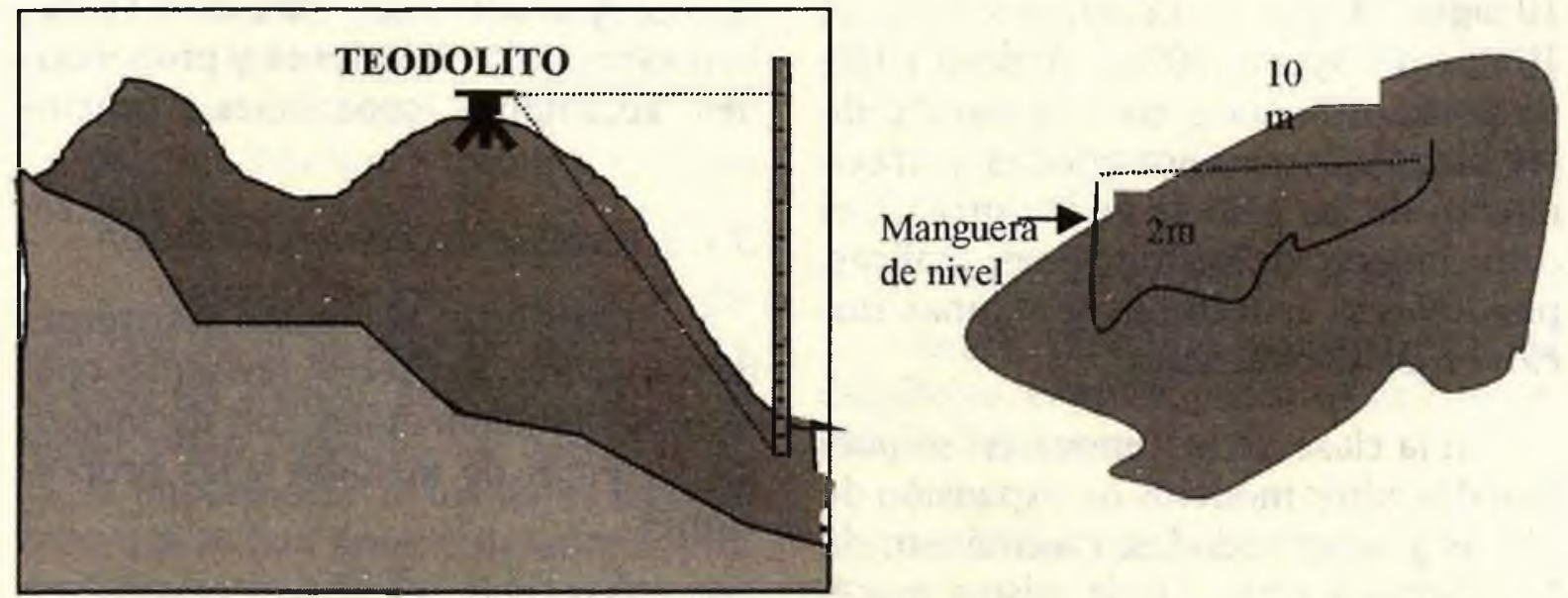

FIGURA 6. Estudio de las pendientes mediante el nivel de mano y el teodolito.

- Disposición de aguas y formas de riego: Trazado de canales para riego, caudales, distribución de aguas y tuberías, variación de caudales. En el programa de matemáticas se puede incluir el estudio de la circunferencia, volúmenes y áreas, nociones de estadística, cálculo de caudales

(caudal $=$ volumen/tiempo), cálculo de laminas de agua, pendientes y funciones.

\subsubsection{Variable de producción.}

- Preparación del terreno: cercos, desinfección y abonados, trazados, semilleros, siembra de sombríos y densidad. Se garantizan buenos cálculos si se comprende la proporcionalidad, representación gráfica de las proporciones, extrapolación e interpolación de resultados, pendientes, porcentajes. Podrían estudiarse fraccionarios a partir de las relaciones entre números de plantas o semillas y unidad de terreno, también entre masa de las semillas y volumen.

Se pueden construir ejemplos como: ¿cuántas libras de semilla se ne-
FIGURA 7. Toma de niveles con manguera.

cesita para sembrar una hectárea a 30 $\mathrm{cm}$ entre planta y a $80 \mathrm{~cm}$ entre surcos, si se sabe que una libra tiene 2000 granos?

Calculemos cuantas plantas caben en la hectárea:

$\mathrm{N}^{\circ}$ de plantas $=(100 \mathrm{~m} / 0,8 \mathrm{~m})$ $(100 \mathrm{~m} / 0,3 \mathrm{~m})=41.666$ granos

luego $41.666 / 1000=41,6$ libras. Por tanto se deben usar aproximadamente 42 libras de semilla.

- Riegos. Estanques, caudales, riegos, cantidad de riegos. Se procura hacer cálculos sobre caudales $(\mathrm{q}=$ volumen/tiempo o velocidad por área transversal), medidas que se pueden hacer en las acequias y ríos. Trate también la conversión de medidas y los máximos y mínimos como aplicación de las derivadas (para maximizar volúmenes).

- Labores culturales. Aporque, control de malezas, plagas y enfermedades, fertilización, podas y raleos. Se requiere la habilidad para el cálculo de dosis y proporción de pesticidas y abonos. Por ejemplo un fertilizante 10-30- 
10 significa que esta compuesto por el $10 \%$ de nitrógeno, $30 \%$ de fósforo y $10 \%$ de potasio. También para el estudio de las plagas, las enfermedades y crecimiento de las plantas es importante el conocimiento de las funciones: gráficas, pendientes, ecuaciones y algunas nociones de estadística.

En la clase de matemáticas se pueden descubrir modelos de expansión de plagas y enfermedades, crecimiento de las plantas y otros. De la misma manera como lo muestra la figura 8 .

- Cosecha. Recolección y/o beneficio y/o desgranado y/o clasificación. El cosechador puede hacer aproximaciones de empaques, obreros, tiempo, ve- hículos y producción. Para esto se deben estudiar las relaciones y proporciones, volúmenes, superficies y funciones.

\subsection{3 variable de comercialización \\ - Transporte. Vehículos y sistemas de transporte. Es preciso revisar las ope- raciones fundamentales con decimales, los sistemas de medidas y las propor- ciones.}

- Mercadeo. Se debe tener en claro los puntos de equilibrio,funciones lineales, cuadráticas y las fracciones. (figura 9).

La gráfica muestra de manera sencilla un estudio sobre el comportamien-

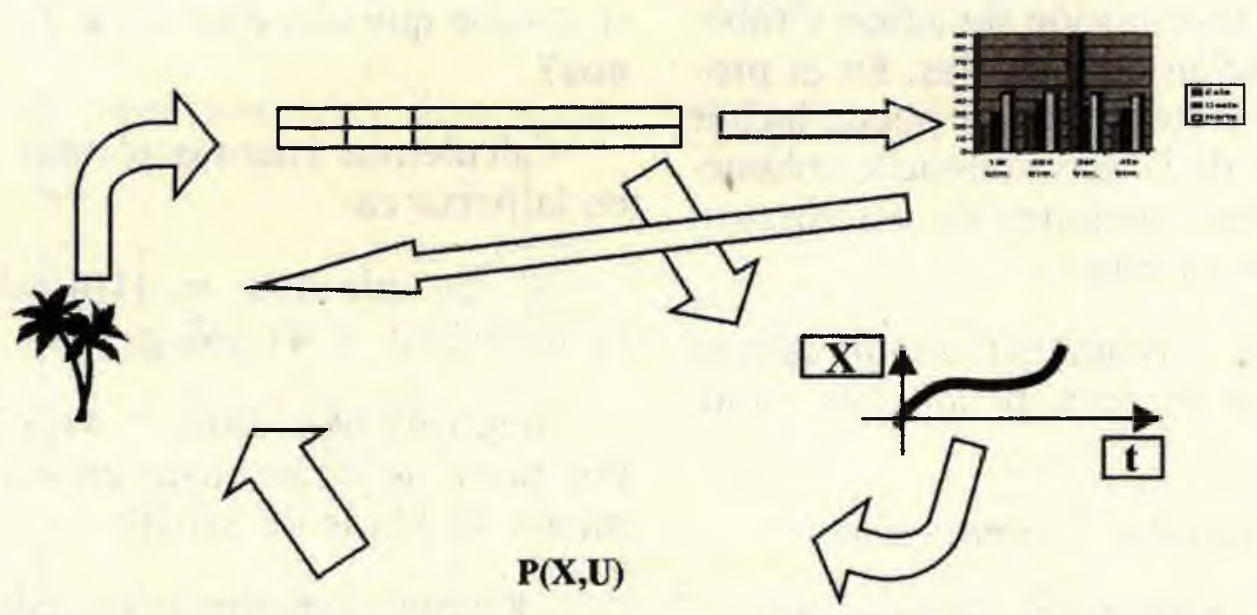

FIGURA 8. Dinámica del procesamiento de la información. Creación de un modelo Matemático. Donde $\mathrm{P}$ son los procesos, $\mathrm{X}$ recoge el vector de variables y $\mathrm{U}$ el vector de parámetros.

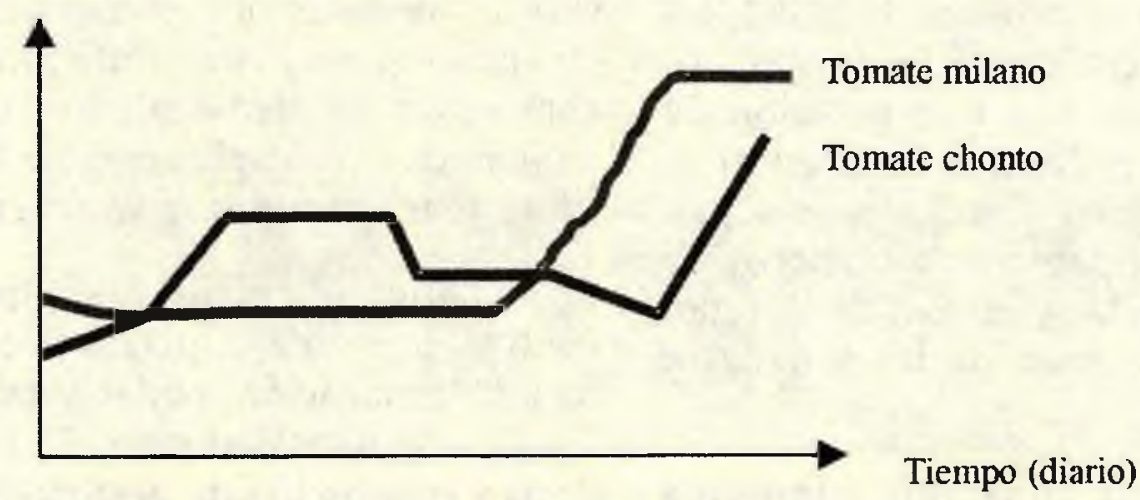

FIGURA 9. Variación del mercado con relación al tiempo. 
to del precio de dos variedades de tomate durante un año, los resultados pueden ser utilizados en la búsqueda de precios buenos y temporadas de máxima producción.

- Cálculo sobre ventas y ganancias. En el análisis de mercado ya se hizo un avance. Sobre los aspectos importantes a tratar solo queda reforzar la importancia de los datos estadísticos que se han llevado durante el proceso productivo y que conjugados con la información de la contabilidad se obtienen buenas aproximaciones sobre las ventas, ganancias, casos de máxima utilidad y mínimo de pérdida.

Todos los aspectos considerados en el presente artículo son aplicables a los cultivos de mediano plazo y perennes, solamente que recomendamos para el caso hacer énfasis en el estudio de la estadística y la contabilidad agrícola para efectuar buenas planeaciones y organizado manejo del proyecto.

Finalmente digamos que el profesor del área de matemáticas puede elaborar su programación basado en el tipo de cultivo que desarrolle el curso o la institución. Tomemos un aparte de la programación para grado octavo que se analizó en la presente tesis para el café:

Area agrícola. Unidad 2

Suelos, trazados, construcción de caballetes, erosión, prácticas de conservación del suelo.

\section{Area de matemáticas}

Construcción de figuras geométricas y teorema de Pitágoras, área y perímetro de triángulos y rectángulos, proporcionalidad, funciones (pendiente de una curva).

Las estrategias y recursos se han venido explicando a lo largo de este trabajo, lo mismo que los criterios de evaluación resultan del proceso seguido.

\section{Resumen}

A continuación se muestra un cuadro que resume los aspectos más importantes del modelo.

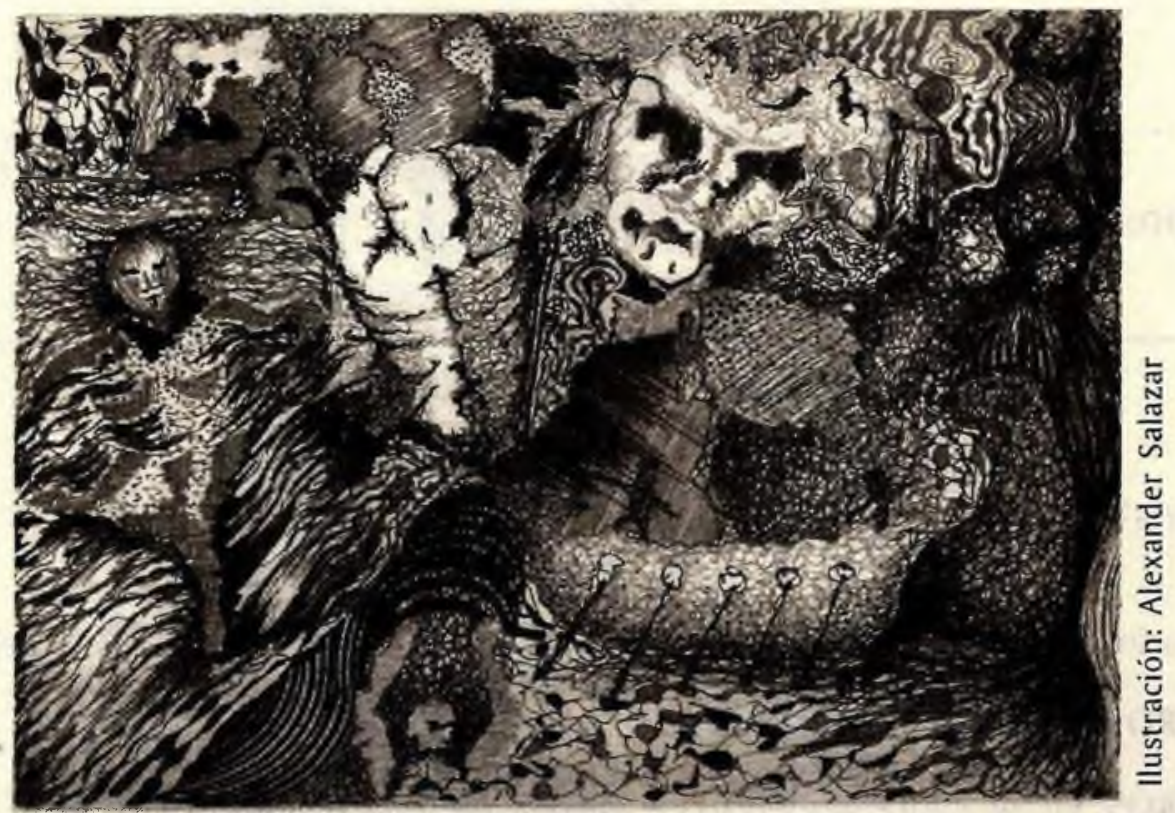


UNIVERSIDAD SURCOLOMBIANA

Cuadro 1.

Muestra aspectos importantes del modelo.

\begin{tabular}{|c|c|c|c|}
\hline VARIABLE & $\begin{array}{l}\text { PARAMETROS Y } \\
\text { RELACIONES }\end{array}$ & CONCEPTOS & SUGERENCIAS \\
\hline $\begin{array}{l}\text { SUELOS } \\
\text { AMBIENTAL }\end{array}$ & $\begin{array}{l}\text { CLIMA } \\
\text { DISPOSICION Y } \\
\text { FORMAS DE RIEGO }\end{array}$ & $\begin{array}{l}\text { Gráficas cartesianas } \\
\text { Areas. volúmenes, tiempo } \\
\text { Figuras geométricas } \\
\text { Máximos y mínimos } \\
\text { Estadística }\end{array}$ & $\begin{array}{l}\text { Medición de caudal } \\
\text { Estudiar crecimien- } \\
\text { to de plagas. } \\
\text { Medir volúmenes en } \\
\text { lagos }\end{array}$ \\
\hline PRODUCCION & $\begin{array}{l}\text { Preparación de terrenos } \\
\text { Semilleros } \\
\text { Siembra } \\
\text { Riegos } \\
\text { Labores culturales } \\
\text { Cosecha }\end{array}$ & $\begin{array}{l}\text { Proporcionalidad } \\
\text { Fraccionarios } \\
\text { Representaciones } \\
\text { Funciones } \\
\text { Derivadas } \\
\text { Sistema de medida } \\
\text { Ecuaciones } \\
\text { Medidas de tendencia } \\
\text { central }\end{array}$ & $\begin{array}{l}\text { Trazado de siembra } \\
\text { Estudio de fertilizantes. } \\
\text { Cálculos sobre terrenos } \\
\text { dosis, mano de obra } \\
\text { cálculos de empaques } \\
\text { densidades. }\end{array}$ \\
\hline COMERCIALIZACION & $\begin{array}{l}\text { Ventas } \\
\text { Ganancias } \\
\text { Campos de } \\
\text { comercialización }\end{array}$ & $\begin{array}{l}\text { Sistemas de medidas } \\
\text { Relaciones } \\
\text { Medidas de tendencia } \\
\text { central } \\
\text { Probabilidades } \\
\text { Contabilidad } \\
\text { Ecuaciones lineales } \\
\text { Proporcionalidad } \\
\text { Operar con reales. }\end{array}$ & $\begin{array}{l}\text { Consultar en internet } \\
\text { Centrales de abastos } \\
\text { Centros de acopio }\end{array}$ \\
\hline PLANEACION & $\begin{array}{l}\text { Presupuesto } \\
\text { Financiación }\end{array}$ & $\begin{array}{l}\text { Programación lineal } \\
\text { Contabilidad }\end{array}$ & $\begin{array}{l}\text { Usar los recursos de la } \\
\text { institución o fincas } \\
\text { cercanas. }\end{array}$ \\
\hline
\end{tabular}

\section{Bibliografía}

AROCA, Carlos. Programación general de producción agrícola. Baraya: Núcleo el Patía, 1998.

BALLESTEROS, Víctor Raúl. Administración de la producción. Bogotá: UNISUR. 1986. 50 p.

BALLESTEROS, Víctor Raúl. Elementos de cálculo diferencial. Bogotá: UNISUR. 1986. 100 p. 
PAIDELA

BERTALAFFY, L.V. Perspectivas en la teoría general de sistemas. Madrid:: Alianza, 1979.

CASTILlEJO, J.L. y COLOM,A.J. Pedagogía sistémica. Barcelona: CEAC, 1995.

COLOM, A. Sociología de la educación y teoría de los sistemas. Barcelona: OIKOS- TAU,S.A. 1978.

LOPEZ, Serafin y RODRIGUEZ Gladys aurora. Tesis de post-grado: Modelo matemático en el currículo del área técnica de colegios agrícolas. Neiva. 1999.

DURAN, Alicia y PEREZ, J.M. La agricultura y el medio rural. Madrid: Paraninfo, 1977.87p.

FIGUEROA, Max y PRIETO, Abel. La escuela secundaria básica en el campo, una innovación educativa en Cuba. París: UNESCO, 1974. 15p.

GRUPO ÁREA DE MATEMATICAS. Programación general de Matemáticas. Algeciras: Núcleo Escolar La Arcadia. 1998.

GRUPO ÁREA DE MATEMATICAS Y CIENCIAS. Programación general de matemáticas y ciencias. Baraya: Colegio Nacional Antonio Baraya. 1998.

GRUPO ÁREA TÉCNICA. Programación general de producción agrícola. Algeciras: Núcleo Escolar La Arcadia.1998.

GRUPO BETA. Proporcionalidad, geometría y semejanza. Madrid: Síntesis, 1990. 65p.

MARTINEZ MEDIANO, J.M. Matemáticas 2. Madrid: Mc Graw Hill. 1993.110p.

MEJIA CASTRO, Benildo. Economia de la empresa. Bogotá: UNISUR. 1986. 20p.

RIOS MEJIA, Aquilino. Curso de Contabilidad. Medellín: Bedout. 1981. 25p.

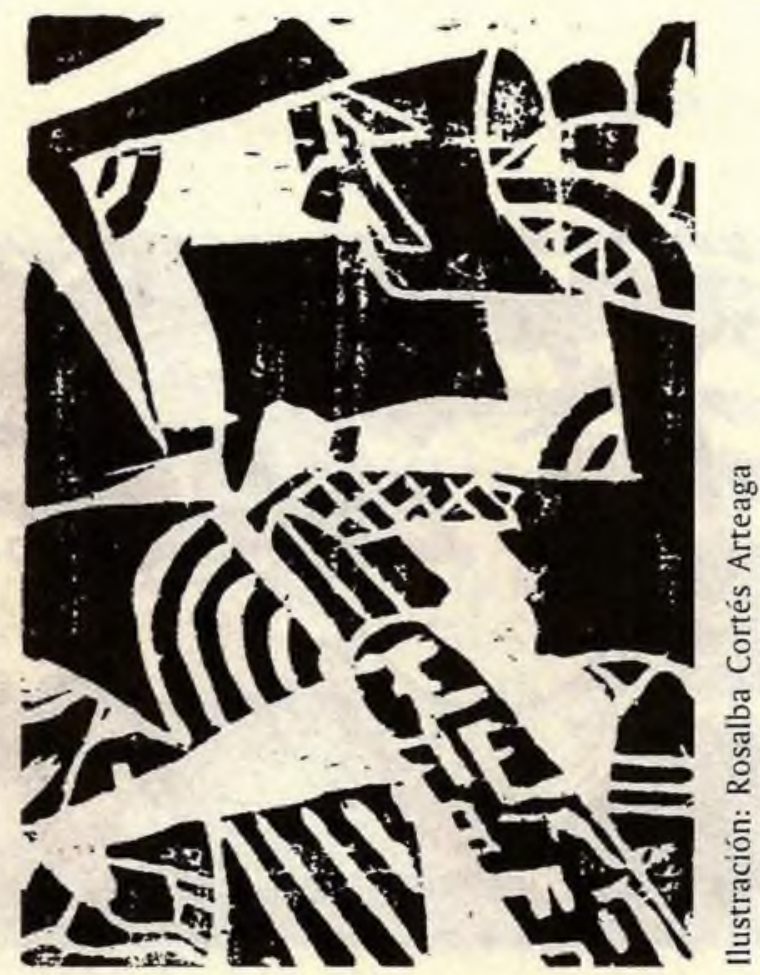


llustración: Luis Fernando Bernal

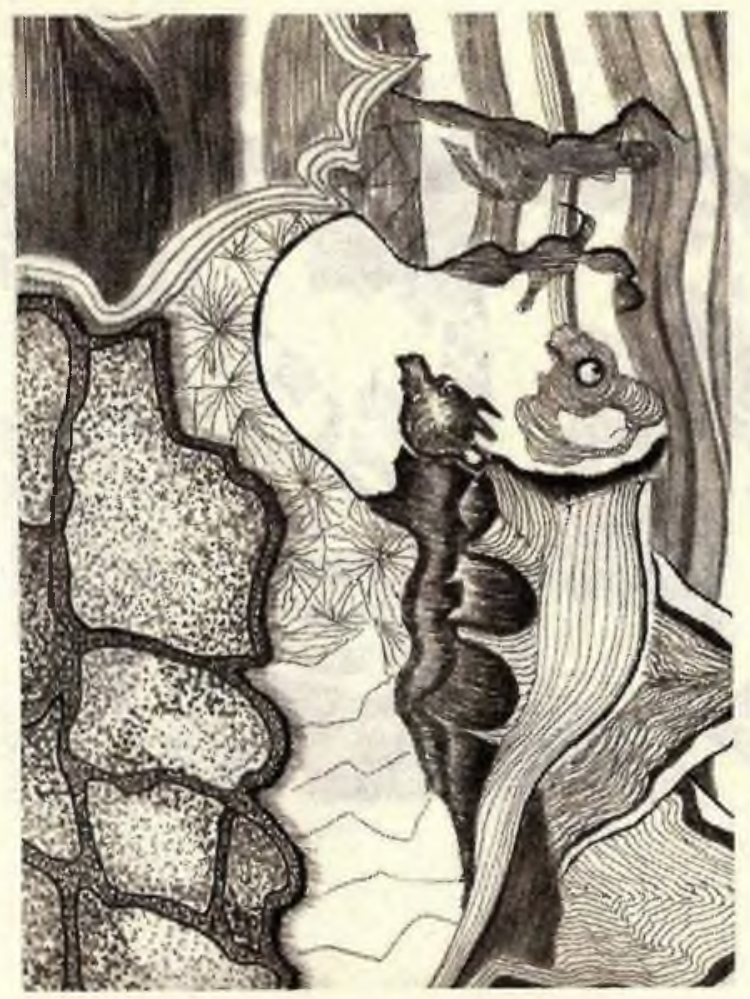


